\title{
Fratura patológica em mandíbula relacionada a lesão cística: relato de caso clínico
}

\author{
Pathological mandible fracture related to cystic lesion: clinical case report \\ Fracture patológica de mandíbula relacionada com lesión quística: reporte de caso clínico
}

\section{Resumo}

As fraturas de mandíbula são frequentemente originadas por traumatismo direto, contudo, podem surgir fraturas patológicas, em função de lesões tumorais ou císticas. Fraturas patológicas de mandíbula são definidas como fraturas que ocorrem em locais onde anteriormente houve um enfraquecimento ósseo por um processo patológico. As causas mais comumente relacionadas são após procedimentos cirúrgicos para a extração de terceiros molares e pela presença de lesões císticas. O principal sinal desse tipo de fratura é a desarticulação dentária. O objetivo deste estudo foi relatar um caso clínico, de uma paciente, do sexo feminino, 61 anos, leucoderma, que apresentava um cisto dentígero de grande extensão associado ao dente 38 incluso e que durante o trans-operatório houve uma fratura patológica. A abordagem cirúrgica é determinada pelo tipo e pela localização da fratura na mandíbula. Deve-se levar em consideração também a sua etiologia, quando relacionadas a cistos deve-se dar enfoque a extensão da lesão e a quantidade de osso remanescente, para considerar o tipo de redução e fixação ideal para cada caso. Em casos que o remanescente ósseo sadio não é suficiente ou está separado por um grande defeito, a ressecção da região envolvida 
pode ser indicada, eventualmente acompanhada de reconstrução imediata ou secundária. Concluiu-se que a redução e a fixação das fraturas mandibulares devem ocorrer tão precisa e rapidamente quanto possível, visto que a maloclusão é uma complicação grave a longo prazo. Além disso, é necessária uma equipe preparada para essa e qualquer tipo de ocorrência durante e após os procedimentos maxilofaciais.

Palavras-chave: Fraturas mandibulares; Fratura patológica; Cisto dentígero.

\begin{abstract}
Mandible fractures are often caused by direct trauma, however, pathological fractures may arise, due to tumor or cystic lesions. Pathological fractures of the mandible are defined as fractures that occur in places where bone has previously been weakened by a pathological process. The most commonly related causes are after surgical procedures to extract third molars and the presence of cystic lesions. The main sign of this type of fracture is tooth disarticulation. The aim of this study was to report a clinical case, of a female patient, 61 years old, leucoderma, who presented a dentigerous cyst of great extension associated with tooth 38 and that during the trans-operative period there was a pathological fracture. The surgical approach is determined by the type and location of the fracture in the mandible. Its etiology should also be taken into account, when related to cysts, the extension of the lesion and the amount of remaining bone should be focused, in order to consider the ideal type of reduction and fixation for each case. In cases where the healthy bone remnant is not sufficient or is separated by a large defect, resection of the involved region may be indicated, possibly accompanied by immediate or secondary reconstruction. It was concluded that reduction and fixation of mandibular fractures should occur as accurately and quickly as possible, as malocclusion is a serious longterm complication. In addition, a team prepared for this and any type of occurrence during and after maxillofacial procedures is needed.
\end{abstract}

Keywords: Mandibular fractures; Pathological fracture; Dentigerous cyst.

\title{
Resumen
}

Las fracturas de la mandíbula a menudo son causadas por un traumatismo directo, sin embargo, pueden surgir fracturas patológicas debido a lesiones tumorales o quísticas. Las fracturas patológicas de la mandíbula se definen como fracturas que ocurren en lugares donde el hueso ha sido previamente debilitado por un proceso patológico. Las causas más comúnmente relacionadas son después de procedimientos quirúrgicos para extraer terceros molares y la presencia de lesiones quísticas. El principal signo de este tipo de fractura es la desarticulación del diente. El objetivo de este estudio fue reportar un caso clínico, de una paciente de sexo femenino, de 61 años, leucoderma, quien presentó un quiste dentígero de gran extensión asociado al diente 38 y que durante el transoperatorio se presentó una fractura patológica. El abordaje quirúrgico está determinado por el tipo y la ubicación de la fractura en la mandíbula. También se debe tener en cuenta su etiología, cuando se relaciona con quistes, se debe enfocar la extensión de la lesión y la cantidad de hueso remanente, con el fin de considerar el tipo de reducción y fijación ideal para cada caso. En los casos en que el remanente óseo sano no sea suficiente o esté separado por un gran defecto, puede estar indicada la resección de la región afectada, posiblemente acompañada de una reconstrucción inmediata o secundaria. Se concluyó que la reducción y fijación de las fracturas mandibulares debe ocurrir con la mayor precisión y rapidez posible, ya que la maloclusión es una complicación grave a largo plazo. Además, se necesita un equipo preparado para esto y cualquier tipo de ocurrencia durante y después de los procedimientos maxilofaciales.

Palabras clave: Fracturas mandibulares; Fractura patológica; Quiste dentígero.

\section{Introdução}

O cisto dentígero é classificado como cisto odontogênico de desenvolvimento, que envolve a coroa de um dente não erupcionado e é caracterizado pelo acúmulo de líquido no espaço entre o epitélio reduzido do esmalte e a coroa do dente. Ocorre com maior frequência em terceiros molares inferiores (Neville et al., 2009; Regezi et al., 2011; Siqueira et al., 2021).

A maioria dos casos são assintomáticos e as lesões são descobertas por exames radiográficos de rotina. Alguns cistos odontogênicos podem ter uma dimensão considerável e causar expansão de cortical óssea e sensibilidade na área envolvida, tendo assim prognóstico desfavorável e complicações como a fratura patológica (Bonardi et al., 2020; Neville et al., 2009; Regezi et al., 2011; Xiao, et al. 2018).

As causas mais comuns de fraturas maxilofaciais, são os traumas causados por acidentes automobilísticos e por agressão física. A presença de patologias ósseas pode causar a redução da resistência à tração e auxiliar na propagação de uma fratura na região enfraquecida, causando o que a literatura aborda como "fratura patológica" (Ezsiás \& Sugar, 1994). Observase também, um número crescente de fraturas decorrentes de queda da própria altura em idosos (Xiao, et al. 2018). 
Fraturas mandibulares decorrentes de lesões císticas são muito raras e com apenas 10 casos relatados na literatura recente, sendo eles relacionados a diferentes patologias como cisto ósseo aneurismático, cisto folicular, cisto residual e ceratocisto odontogênico (Algahtani et al., 2009; Coletti \& Ord, 2008; Choi et al., 2011; Gerhards et al., 1998).

As fraturas patológicas de mandíbula são definidas como fraturas que ocorrem em locais onde anteriormente houve um enfraquecimento ósseo por um processo patológico. As causas mais comumente relacionadas são após procedimentos cirúrgicos para a extração de terceiros molares ou instalação de implantes, facilitadas pela presença de lesões císticas e tumorais, decorrentes de osteomielite, osteorradionecrose e osteonecrose induzidas pelo uso de bifosfonatos (Algahtani et al., 2009; Bonardi et al., 2020; Coletti \& Ord, 2008; Choi et al., 2011; Gerhards et al., 1998).

Os sinais e sintomas relacionados às fraturas patológicas causadas por cistos radiculares abrangem tanto hiperalgesia como hipoalgesia, inchaço e equimose (Pimentel, et al. 2021; Xiao, et al. 2018). O sinal patognomônico mais comum da fratura mandibular é o "desarticulado interdental" ou a oclusão dentária. O trismo mandibular também é um sinal frequentemente encontrado (Bonardi et al., 2020; Silva et al, 2011).

Os exames de imagem são de extrema importância para o correto diagnóstico e definição da conduta a ser tomada. Podemos utilizar desde a radiografia panorâmica a tomografia computadorizada, sendo a tomografia computadorizada o padrão ouro, pois gera maior detalhes e dimensões, como impactação de estruturas nobres (Lima et al, 2017; Silva et al, 2011).

O tratamento das fraturas patológicas é determinado de acordo com sua etiologia, quando relacionadas a cistos devese dar enfoque a extensão da lesão e a quantidade de osso remanescente, para considerar o tipo de redução e fixação ideal para cada caso (Xiao, et al. 2018; Zavlin, et al. 2018). Em casos que o remanescente ósseo sadio não é suficiente ou está separado por um grande defeito, a ressecção da região envolvida pode ser indicada, eventualmente acompanhada de reconstrução imediata ou secundária, para evitar a fratura indesejada (Coletti \& Ord, 2008; Siqueira et al., 2021).

O propósito do tratamento das fraturas é a redução, podendo ser de forma aberta ou fechada, podendo ser tratadas com fixação interna ou externa onde os tratamentos mais conservadores com técnicas de fixação externas são possíveis. Porém, em sua maioria, é necessário a utilização de fixações internas (Lima et al, 2017; Zavlin, et al. 2018).

O objetivo deste trabalho é relatar um caso clínico, de uma paciente, do sexo feminino, 61 anos, leucoderma, que apresentava um cisto dentígero de grande extensão associado ao dente 38 incluso e que durante o trans-operatório houve uma fratura patológica.

\section{Metodologia}

Refere-se a um relato de caso, caracterizado como um estudo exploratório, descritivo e qualitativo (Pereira et al., 2018).

Foram seguidos os princípios éticos de pesquisa envolvendo seres humanos, sendo preservada a identidade do voluntário e fornecido todos os esclarecimentos sobre o tratamento e após concordância do paciente a assinatura do Termo de Consentimento Livre Esclarecido (TCLE), consentindo o tratamento, a divulgação das imagens e dados coletados durante o atendimento clínico e os que constavam no prontuário, sendo que o mesmo poderia desistir da participação na pesquisa a qualquer instante.

\section{Caso Clínico}

Paciente gênero feminino, 61 anos de idade, leucoderma, compareceu ao ambulatório do Serviço de Cirurgia e Traumatologia Buco-maxilo-facial, após ser encaminhada por outro profissional devido a descoberta de lesão em exame de rotina. A mesma possuía histórico de fístula intra-oral que estava drenando espontaneamente. 
Durante a anamnese, relatou que havia um 'gosto ruim na boca, atrás do último dente", negava ser tabagista e etilista. Estava em acompanhamento médico devido a hipertensão e diabetes. A paciente queixa-se também de sensação dolorosa, frequentes episódios de restrição de abertura de boca, secreção purulenta e odor, que a incomodava.

Ao exame clínico extra-oral, não foi observado nenhuma alteração. No exame intraoral, constatou-se a ausência clínica da unidade dentária 38. Não apresentava aumento de volume ou abaulamento das corticais, pele e mucosa adjacentes normocoradas e ausência de drenagem purulenta (Figura 1).

Figura 1: Exame intra-oral dentro dos padrões de normalidade.

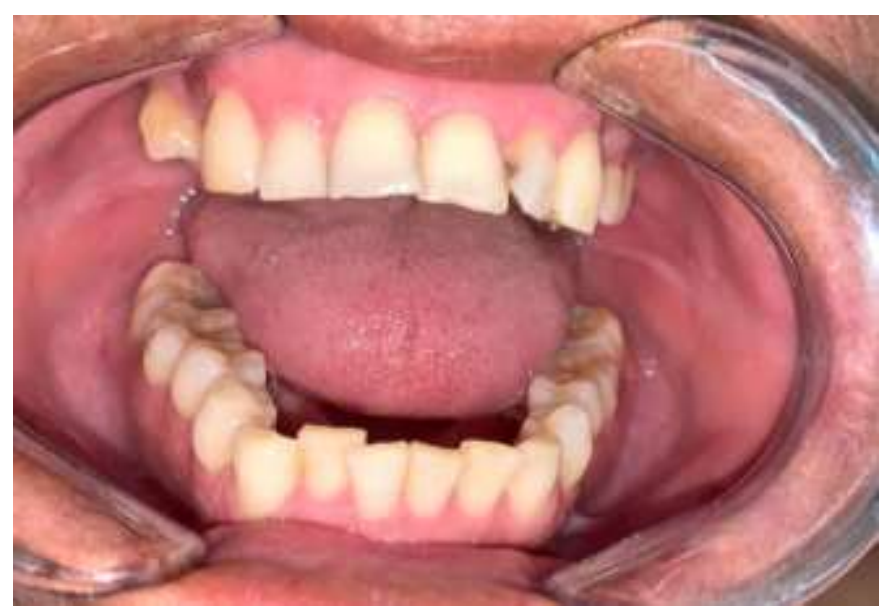

Fonte: Autores.

A radiografia panorâmica evidenciou uma imagem radiolúcida unilocular com limites bem definidos em região posterior de mandíbula esquerda associada à coroa da unidade dentária 38 incluso com aproximadamente $4 \mathrm{~cm}$ de diâmetro (Figura 2). Na tomografia computadorizada de face observou-se uma lesão osteolítica hipodensa em região posterior de mandíbula associada ao dente 38, sem abaulamento ou rompimento das corticais lingual e vestibular. Logo, solicitou-se um exame tomográfico para planejamento do tratamento.

Figura 2: Radiografia panorâmica evidenciando lesão radiolúcida associada ao elemento 38 incluso.

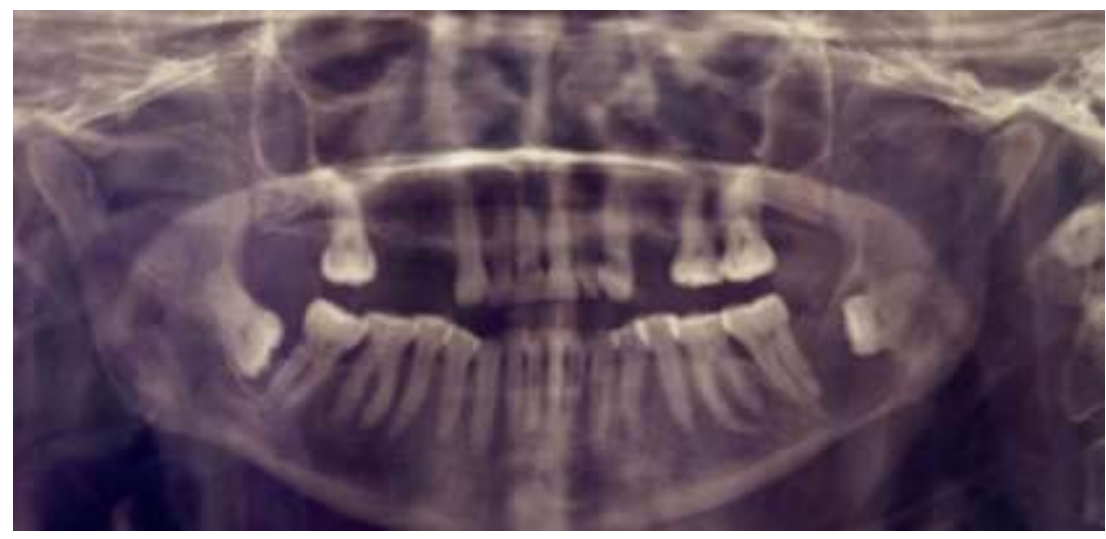

Fonte: Autores. 
A abordagem cirúrgica foi por meio de enucleação seguida de remoção do dente envolvido sob anestesia geral, por meio de um acesso intra-oral (Figura 3). Realizou-se a remoção completa da lesão e durante o trans-operatório houve a fratura patológica na região inferior esquerda (Figura 4). Foi realizada ainda, a biópsia incisional para confirmação do diagnóstico.

Figura 3: Acesso e exposição ao leito patológico.

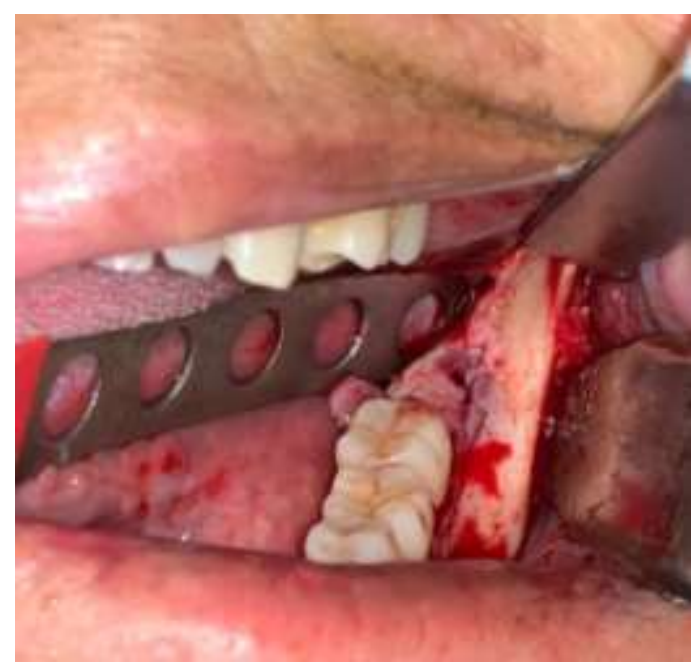

Fonte: Autores.

Figura 4: Leito cirúrgico após a remoção completa da lesão cística.

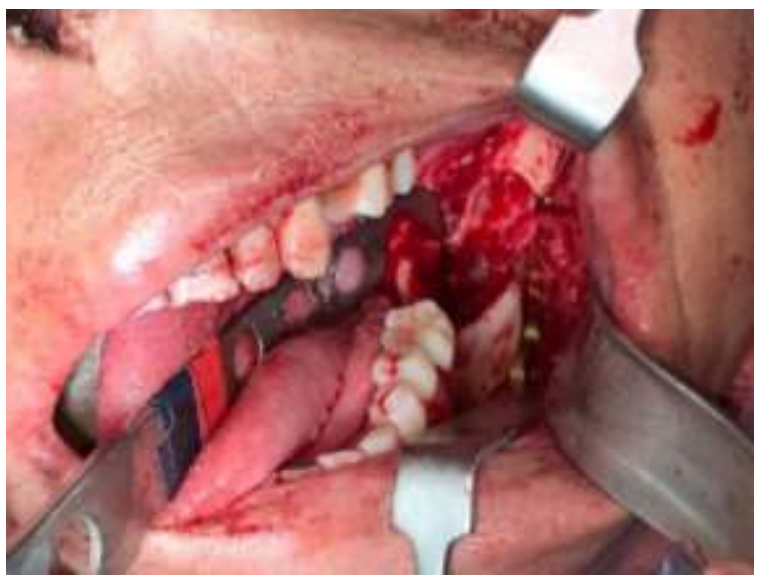

Fonte: Autores.

Diante dessa ocorrência, optou-se pela abordagem cirúrgica por meio de acesso extra-oral de Risdon para ideal visualização do campo operatório e fixação. Devido a extensão da lesão sabia-se da probabilidade de ocorrer essa intercorrência, estando a equipe planejada para lidar com tal acontecimento. Sendo assim, optou-se pela utilização de placa de reconstrução $2.4 \mathrm{~mm}$ load bearing, na região de ramo e ângulo mandíbular (Figura 5). 
Figura 5: Observa-se por acesso extra-oral a placa adaptada na região fraturada.

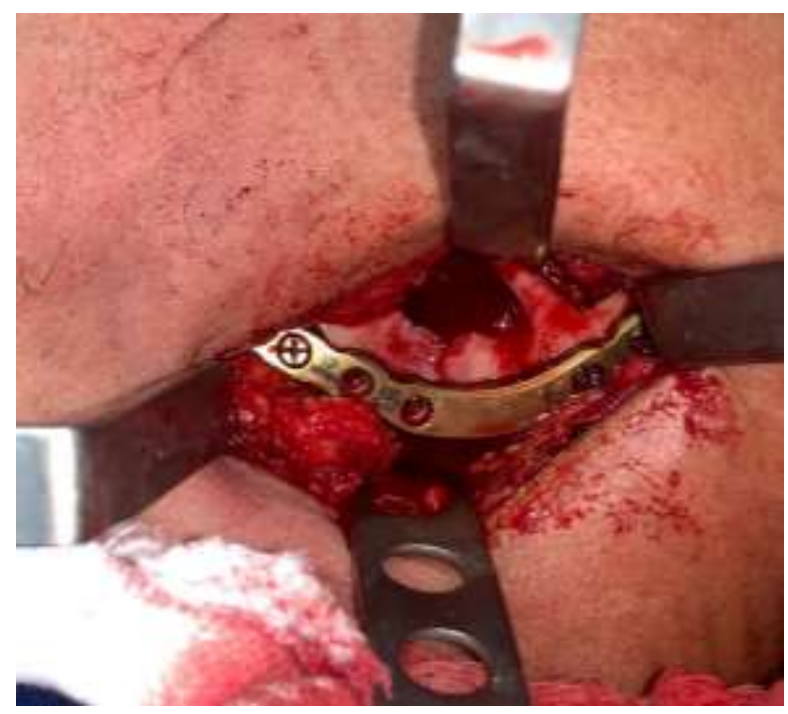

Fonte: Autores.

O resultado da biópsia revelou fragmentos de parede fibrosa sede de marcante tecido de granulação e inflamação linfoplasmoscitária predominantemente, ao lado de fendas de colesterol e reação gigantocelular tipo corpo estranho, focos de hemácias recentes e antigas, revestida por epitélio escamoso estratificado exibindo acantose sem atipias e exocitose neutrofílica. Outras secções mostram dentes de esboço usual, mas com remanescentes de matriz de esmalte, osso cortical e regional bem diferenciado permeado por tecido estromal fibrovascular. O diagnóstico foi de cisto dentígero.

A radiografia panorâmica e PA de face mostram correta adaptação e redução anatômica da área fraturada no pósoperatório imediato (Figura 6).

Figura 6: Radiografia panorâmica do pós-operatório imediato.

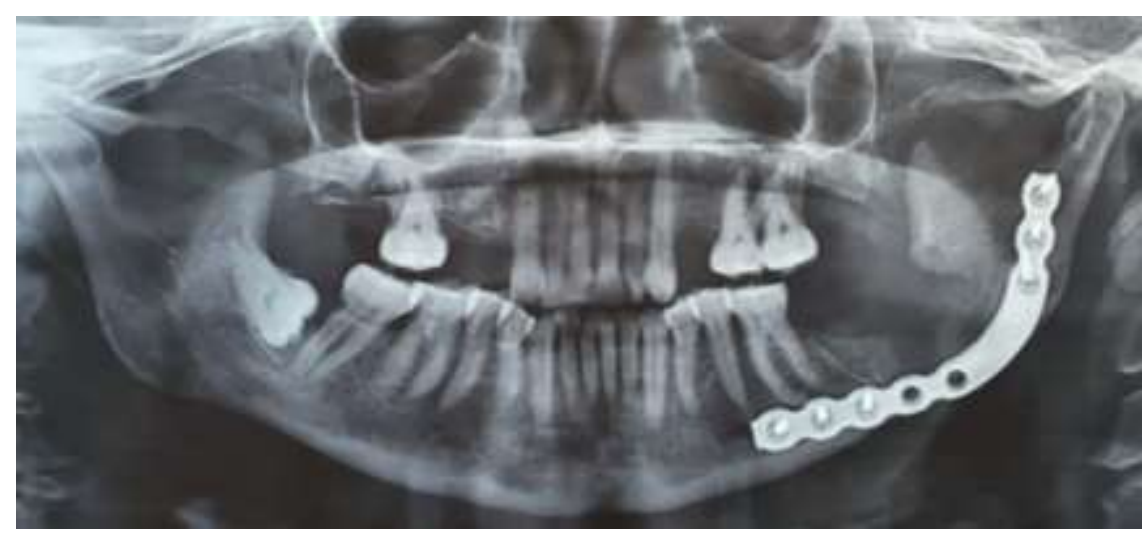

Fonte: Autores.

Não houve lesão permanente no nervo marginal da mandíbula esquerdo e nem ao nervo alveolar inferior esquerdo. A paciente encontra-se em acompanhamento e não há nenhuma complicação até o momento. A cicatrização ocorreu dentro dos padrões de normalidade. Segundo a paciente, não há qualquer sinal de desconforto na área e não sente mais dificuldade de abertura de boca conforme relatou na anamnese (Figura 7). 
Figura 7: Oclusão pós-operatória 1 anos depois.

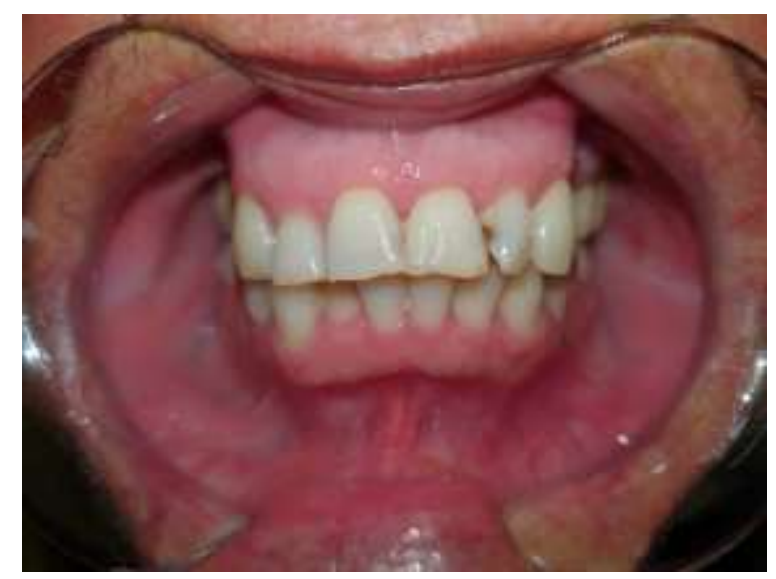

Fonte: Autores.

\section{Discussão}

As fraturas de mandíbula são frequentemente causadas por traumatismo direto, mas, eventualmente, podem surgir fraturas patológicas, em função de lesões tumorais. São consideradas fraturas raras, representando apenas $2 \%$ de todas as fraturas de mandíbula (Algahtani et al., 2009; Bonardi et al., 2020; Coletti \& Ord, 2008; Choi et al., 2011; Gerhards et al., 1998).

A faixa etária mais acometida foi de 50 a 70 anos de idade. A literatura aponta que a faixa etária de 60 anos é a mais acometida nos traumatismos faciais, pelo fato que os idosos são mais propensos a fratura patológica, devido a fragilidade óssea e fatores sistêmicos que influenciam (Boffano et al.; 2013; Coletti \& Ord, 2008). A paciente soma-se a literatura, visto que ela possui 61 anos.

As regiões anatômicas mais acometidas com fraturas patológicas foram ângulo e corpo de mandíbula (Ezsias \& Sugar, 1994; Goddard \& Patel, 2007; Kalantar, 1998). Segundo Neville et al. 2009, o local mais comumente atingido é a parassínfise. O presente relato revelou a fratura em região de ângulo mandibular.

São mais frequentemente causadas por osteorradionecrose, e os cistos, mesmo que sejam comuns em região maxilofacial, muito raramente causam fraturas (Boffano et al.; 2013; Coletti \& Ord, 2008; Ezsia \& Sugar, 1994; Gerhards et al., 1998). Neste estudo, a fratura ocorreu devido a fragilidade causada pelo cisto e durante a remoção, ocorreu a intercorrência.

O sinal clássico dessa classe de fratura é a dor e má oclusão (Goddard \& Patel, 2007; Silva et al, 2011; Siqueira et al., 2021). A paciente em questão apresentava-se apenas com dor e não havia má oclusão, porém, queixava-se de trismo.

Nos casos em que há um bom suporte ósseo para a fratura, é recomendado realizar a redução aberta e fixação interna, em associação com a enucleação ou marsupialização da lesão cística (Boffano et al.; 2013; Ezsias \& Sugar, 1994; Goddard \& Patel, 2007; Kalantar, 1998).

Geralmente ocorre antes da remoção da lesão patológica, devido ao potencial de causar áreas de reabsorção óssea que essas podem apresentar, levando a uma região enfraquecida e propícia a fratura (Ezsias \& Sugar, 1994; Goddard \& Patel, 2007; Kalantar, 1998). Neste caso, ocorreu durante a remoção.

\section{Conclusão}

Por fim, concluiu-se que é válido ressaltar a importância do conhecimento quanto as alterações patológicas que acometem a cavidade oral, para que seja realizado o diagnóstico correto e consequente o tratamento adequado. O diagnóstico 
deve ser estabelecido por exame clínico e radiográfico. O sinal clínico bastante evidente da fratura patológica é a má oclusão, que por vezes está acompanhada de desconforto na área afetada.

A conduta cirúrgica é determinada pelo tipo, pela localização da fratura e quantidade de osso remanescente, para considerar o tipo de redução e fixação ideal para cada caso. A técnica cirúrgica empregada deve ser segura tomando os devidos cuidados. É necessário planejamento cirúrgico cauteloso para remoção da lesão, levando em consideração a extensão da lesão, relação do cisto com estruturas anatômicas importantes e como neste caso, as possíveis complicações que o ato cirúrgico pode ocasionar.

Ademais, para os próximos trabalhos, sugere-se que sejam relatados novos casos de fratura patológica em mandíbula e o tipo de tratamento empregado, visto que a literatura careca de informações a respeito do tema.

\section{Referências}

Algahtani, M., Alqudah, M., Alshehri, S., Binahmed, A. \& Sandor, GK. (2009). Pathologic fracture of the mandible caused by metastatic follicular thyroid carcinoma. J Can Dent Assoc, 75(6), 457-60.

Boffano, P., Roccia, F., Gallesio, C. \& Berrone, S. (2013). Pathological mandibular fractures: a review of the literature of the last two decades. Dental Traumatology, 29(3), 185-96.

Bonardi, J. P., Momesso, G. A. C., Lima, V. N. de., Santos, A. M. de S., Rios, B. R., Reis, E. N. R. de C., Silva, L. de F., Vieira. E. H., Santiago Júnior, J. F., \& Faverani, L. P. (2020). Etiological factors for mandibular fractures in transoperative period of tooth extraction: systematic review. Research, Society and Development, $9(9)$.

Coletti, D., \& Ord, R. A. (2008). Treatment rationale for pathological fractures of the mandible: a series of 44 fractures. Int J Oral Maxillofac Surg, 37(3), $215-22$.

Choi, BJ., Choi, S. C. \& Kwon, Y. D. (2011). Aneurysmal bone cyst causing a pathologic fracture of the mandibular condyle. J Oral Maxillofac Surg, 69(12), 2995-3000.

Ezsias, A. \& Sugar, AW. (1994). Pathological fractures of the mandible: a diagnostic and treatment dilemma. Br J Oral Maxillofac Surg, 32(5), 303-6.

Gerhards, F., Kuffner, HD. \& Wagner, W. (1998). Pathological fractures of the mandible. A review of the etiology and treatment. Int J Oral Maxillofac Surg, 27(3), 186-90.

Goddard, R. \& Patel, N. (2007). Aneurysmal bone cyst masquerading as unknown mandibular metastatic deposit causing pathological fracture. Dent Update, 34(4), 230-2.

Lima, V, N., Figueiredo, C, M, B, F., Momesso, G, A, C., Queiros, S, B, F., \& Faverani, L, P. (2017). Fratura mandibular associado à remoção de terceiro molar inferior: revisão de literatura. Arch Health Invest 6(9):414-417.

Kalantar, M., Motamedi M. H. (1998). Aneurysmal bone cysts of the jaws: clinicopathological features, radiographic evaluation and treatment analysis of 17 cases. J Craniomaxillofac Surg, 26(1), 56-62.

Pereira, A. S., Shitsuka, D. M., Parreira, F. J. \& Shitsuka, R. (2018). Metodologia da pesquisa científicaUFSM.https://repositorio.ufsm.br/bitstream/handle/1/15824/LicComputacao_Metodologia-Pesquisa-Cientifica.pdf?sequence=1.

Pimentel, T., Hadad, H., Jesus, L. K., Colombo, L. T., Alcantara, A. G., Souza, F. Á., \& Gárcia-Júnior, I. R. (2021). Pathological fracture of the jaw due to osteomyelitis after a third molar extraction. Archives of health investigation, 10(4), 637-640.

Neville, B. W., Damm, D. D., Allen, C. M. \& Bouquot, JE. (2009). Oral and maxillofacial pathology, (3rd ed.), Saunders Elsevier. 678-731.

Regezi, J., A., Sciubba, J. J. \& Jordan, R. C. K. (2011). Oral pathology: clinical pathologic correlations, (6th ed.), Saunders Elsevier. $237-51$.

Siqueira, N. B. de, Costa Filho, J. R. T., Rodrigues, J. V. S., Hochuli-Vieira, E., Okamoto, R., \& Vasconcelos, B. C. do E. (2021). Unusual treatment for calcifying odontogenic cyst using decompression tube to prevent pathological fracture. Research, Society and Development, 10(1),

Silva, J. J. D. L., Lima, A. A. A. S., Dantas, T. B., Frota, M. H. A. D., Parente, R. V., \& Lucena, A. L. S. P. D. N. (2011). Fratura de mandíbula: estudo epidemiológico de 70 casos. Revista Brasileira de Cirurgia Plástica, 26(4), 645-648.

Zavlin, D., Jubbal, K. T., Echo, A., Izaddoost, S. A., Friedman, J. D., \& Olorunnipa, O. (2018). Multi-institutional analysis of surgical management and outcomes of mandibular fracture repair in adults. Craniomaxillofacial trauma \& reconstruction, 11(01), 041-048.

Xiao, X., Dai, J. W., Li, Z., \& Zhang, W. (2018). Pathological fracture of the mandible caused by radicular cyst: A case report and literature review. Medicine, 97(50), 1-4. 